\title{
ON GENERALISED CONVEX MULTI-OBJECTIVE NONSMOOTH PROGRAMMING
}

\author{
S. K. MISHRA and R. N. MUKHERJEE ${ }^{1}$
}

(Received 15 February 1994; revised 21 July 1994)

\begin{abstract}
We extend the concept of $V$-pseudo-invexity and $V$-quasi-invexity of multi-objective programming to the case of nonsmooth multi-objective programming problems. The generalised subgradient Kuhn-Tucker conditions are shown to be sufficient for a weak minimum of a multi-objective programming problem under certain assumptions. Duality results are also obtained.
\end{abstract}

\section{Introduction}

In the differentiable case, Jeyakumar and Mond [3] defined a vector invexity that avoids the major difficulty of verifying that the inequality holds for the same function $\eta(\cdot, \cdot)$ for invex functions. Jeyakumar and Mond [3] established sufficient optimality criteria under $V$-pseudo-invexity and $V$-quasi-invexity and obtained duality results under these assumptions. This relaxation allows us to treat nonlinear fractional programming problems also. Egudo and Hanson [2] used the concept of Zhao [4] to generalise the concept of $V$-invexity of Jeyakumar and Mond [3] to the nonsmooth case by replacing the gradients with the gradients of Clarke [1].

In this paper we extend the concept of $V$-pseudo-invexity and $V$-quasi-invexity of Jeyakumar and Mond [3] to the nonsmooth case. Further sufficient optimality conditions and duality results have been derived for such nonsmooth multi-objective programming.

${ }^{1}$ Dept of Applied Math, Inst. of Technology, Banaras Hindu University, Varanasi 221005, India.

(C) Australian Mathematical Society, 1996, Serial-fee code 0334-2700/96 


\section{Preliminaries}

Egudo and Hanson [2] considered the nonlinear multi-objective programming problem:

$$
\begin{array}{ll}
\text { Minimise } & \left(f_{i}(x) ; i=1,2, \ldots, p\right) \\
\text { subject to } & g_{j}(x) \leq 0, j=1,2, \ldots, m
\end{array}
$$

where $f_{i}: R^{n} \rightarrow R, i=1,2, \ldots, p$ and $g_{j}: R^{n} \rightarrow R, j=1,2, \ldots, m$ are locally Lipschitz functions.

The generalised directional derivative of a Lipschitz function $f$ at $x$ in the direction $d$ denoted by $f^{0}(x ; d)$ (see, for example, Clarke [1]) is :

$$
f^{0}(x ; d)=\lim _{\substack{y \rightarrow x \\ t \downarrow 0}} \sup t^{-1}(f(y+t d)-f(y)) .
$$

The Clarke generalised subgradient of $f$ at $x$ is denoted by

$$
\partial f(x)=\left\{\xi: f^{0}(x ; d) \geq \xi^{T} d, \forall d \in R^{n}\right\} .
$$

Egudo and Hanson [2] defined invexity for locally Lipschitz functions as follows. A locally Lipschitz function $f(x)$ is invex on $X_{0} \subset R^{n}$ if for $x, u \in X_{0}$ there exists a function $\eta(x, u): X_{0} \times X_{0} \rightarrow R$ such that $f(x)-f(u) \geq \xi^{T} \eta(x, u), \forall \xi \in \partial f(u)$.

The following example is from [2].

$$
f(x)=\left\{\begin{array}{lll}
20-x & \text { if } \quad x \leq-15 \\
5-2 x & \text { if } \quad-15 \leq x \leq 0 \\
5+2 x & \text { if } \quad 0 \leq x \leq 15 \\
20+x & \text { if } \quad x \geq 15
\end{array}\right.
$$

The function $f(x)$ is regular in the sense of Clarke [1] in that $f^{0}(x ; d)=f^{\prime}(x ; d)$, where $f^{\prime}(x ; d)$ is the directional derivative

$$
f^{\prime}(x ; d)=\lim _{t \downarrow 0} t^{-1}(f(x+t d)-f(x)) .
$$

It was shown in [2] that $f(x)$ is invex.

A locally Lipschitz $f(x)$ is pseudo-invex on $X_{0} \subset R^{n}$ if for $x, u \in X_{0}$ there exists a function $\eta(x, u): X_{0} \times X_{0} \rightarrow R$ such that $\xi^{T} \eta(x, u) \geq 0 \Rightarrow f(x) \geq f(u)$, $\forall \xi \in \partial f(u)$.

A locally Lipschitz $f(x)$ is quasi-invex on $X_{0} \subset R^{n}$ if for $x, u \in X_{0}$ there exists a function $\eta(x, u): X_{0} \times X_{0} \rightarrow R$ such that $f(x) \leq f(u) \Rightarrow \xi^{T} \eta(x, u) \leq 0$, $\forall \xi \in \partial f(u)$.

It is clear from the definitions that every locally Lipschitz invex function is locally Lipschitz pseudo-invex and locally Lipschitz quasi-invex. Examples can be constructed easily. 


\section{Generalised invex vector functions}

In the differentiable case Jeyakumar and Mond [3] defined vector invexity thus: (P) is said to be $V$-invex if there exist $\eta: X_{0} \times X_{0} \rightarrow R^{n}$ and $\alpha_{i}, \beta_{j}: X_{0} \times X_{0} \rightarrow R^{+} \backslash\{0\}$ such that

$$
\begin{aligned}
& f_{i}(x)-f_{i}(u)-\alpha_{i}(x, u) \nabla f_{i}(u) \eta(x, u) \geq 0 \\
& g_{j}(x)-g_{j}(u)-\beta_{j}(x, u) \nabla g_{j}(u) \eta(x, u) \geq 0 .
\end{aligned}
$$

Jeyakumar and Mond [3] further extended $V$-invexity to $V$-pseudo-invexity and $V$ quasi-invexity.

Using the results of Zhao [4], Egudo and Hanson [2] generalised the $V$-invexity concept of Jeyakumar and Mond [3] to the nonsmooth case by replacing the gradients $\nabla f_{i}$ and $\nabla g_{j}$ with the generalised gradients of Clarke [1]. Hence (P) is said to be $V$-invex if there exist $\eta: X_{0} \times X_{0} \rightarrow R^{n}$ and $\alpha_{i}, \beta_{j}: X_{0} \times X_{0} \rightarrow R^{+} \backslash\{0\}$ such that

$$
\begin{array}{ll}
f_{i}(x)-f_{i}(u)-\alpha_{i}(x, u) \xi_{i} \eta(x, u) \geq 0, & \forall \xi_{i} \in \partial f_{i}(u), \\
g_{j}(x)-g_{j}(u)-\beta_{j}(x, u) \zeta_{j} \eta(x, u) \geq 0, & \forall \zeta_{j} \in \partial g_{j}(u)
\end{array}
$$

The following example is a $V$-invex nonsmooth multi-objective programming problem. Consider the multi-objective problem

$$
V \text {-minimise } \quad\left(\left|\frac{2 x_{1}-x_{2}}{x_{1}+x_{2}}\right|, \frac{x_{1}+2 x_{2}}{x_{1}+x_{2}}\right)
$$

subject to $x_{1}-x_{2} \leq 0,1-x_{1} \leq 0,1-x_{2} \leq 0, \alpha_{i}(x, u)=1$ for $i=1,2$, $\beta_{j}(x, u)=\left(x_{1}+x_{2}\right) / 3$ for $j=1,2$ and

$$
\eta_{i}(x, u)=\left(\frac{3\left(x_{1}-1\right)}{x_{1}+x_{2}}, \frac{2\left(x_{2}-2\right)}{x_{1}+x_{2}}\right)^{\top} .
$$

As we can see the generalised directional derivative of $f_{1}(x)=\left|\frac{2 x_{1}-x_{2}}{x_{1}+x_{2}}\right|$ is

$$
\begin{aligned}
f^{0}(x ; d) & =\limsup _{\substack{y_{1} \rightarrow x_{1} \\
t \downarrow 0}} t^{-1}\left[\left|\frac{2\left(y_{1}+t d\right)-x_{2}}{y_{1}+t d+x_{2}}\right|-\left|\frac{2 y_{1}-x_{2}}{y_{1}+x_{2}}\right|\right] \\
& \left.=\limsup _{\substack{y_{1} \rightarrow x_{1} \\
t \downarrow 0}} t^{-1}\left[\frac{3 t d x_{2}}{\left(y_{1}+x_{2}+t d\right)\left(y_{1}+x_{2}\right)}\right] \text { (if } \frac{2 x_{1}-x_{2}}{x_{1}+x_{2}} \geq 0\right) \\
& =\frac{3 d x_{2}}{\left(x_{1}+x_{2}\right)^{2}} .
\end{aligned}
$$


If we take $x_{1}=1$ and $x_{2}=2$ (that is, for an efficient solution $(1,2)$ ) then $f^{0}(x ; d)=2 d / 3$.

If $y_{2} \rightarrow x_{2}$, then $f^{0}(x ; d)=-d / 3$. Thus $(2 d / 3,-d / 3) \in \partial f_{1}(u)$. It is easy to see that $(-2 / 9,1 / 9) \in \partial f_{2}(u)$. At these particular points we can easily see that the above program is $V$-invex for the nonsmooth case.

We now extend $V$-invexity as in Egudo and Hanson [2] to $V$-pseudo-invexity and $V$-quasi-invexity.

A vector function $f: X_{0} \rightarrow R^{P}$ is said to be $V$-pseudo-invex if there exist functions $\eta: X_{0} \times X_{0} \rightarrow R^{P}$ and $\alpha_{i}: X_{0} \times X_{0} \rightarrow R_{+} \backslash\{0\}$ such that for each $x, u \in X_{0}$,

$$
\sum_{i=1}^{p} \xi_{i} \eta(x, u) \geq 0 \Rightarrow \sum_{i=1}^{p} \alpha_{i}(x, u) f_{i}(x) \geq \sum_{i=1}^{p} \alpha_{i}(x, u) f(u), \quad \forall \xi_{i} \in \partial f_{i}(u) .
$$

The vector function $f$ is said to be $V$-quasi-invex if there exist functions $\eta$ : $X_{0} \times X_{0} \rightarrow R^{p}$ and $\beta_{i}: X_{0} \times X_{0} \rightarrow R_{+} \backslash\{0\}$ such that for each $x, u \in X_{0}$,

$$
\begin{aligned}
\sum_{i=1}^{p} \beta_{i}(x, u) f_{i}(x) & \leq \sum_{i=1}^{p} \beta_{i}(x, u) f_{i}(u) \\
& \Rightarrow \sum_{i=1}^{p} \zeta_{i} \eta(x, u) \leq 0, \quad \forall \zeta_{i} \in \partial f_{i}(u) .
\end{aligned}
$$

It is apparent from the definitions that every $V$-invex function of Egudo and Hanson [2] is $V$-pseudo-invex and $V$-quasi-invex as defined above.

Recall from Jeyakumar and Mond [3] that $u \in X_{0}$ is said to be a (global) weak minimum of a vector function $f: X_{0} \rightarrow R^{p}$ if there exists no $x \in X^{0}$ for which $f_{i}(x)<f_{i}(u), i=1, \ldots, p$.

\section{Sufficiency and duality}

In this section we show that the subgradient Kuhn-Tucker conditions are sufficient for a weak minimum in $(\mathrm{P})$ when generalised $V$-invexity is present.

THEOREM 4.1. Let $(u, \tau, \lambda)$ satisfy the Kuhn-Tucker conditions that

$$
\begin{gathered}
0 \in \sum_{i=1}^{p} \tau_{i} \partial f_{i}(u)+\sum_{j=1}^{m} \lambda_{j} \partial g_{j}(u), \quad \lambda_{j} g_{j}(u)=0, \quad j=1,2, \ldots, m, \\
\tau_{i} \geq 0, \quad \tau^{T} e>0, \quad y_{i} \geq 0 .
\end{gathered}
$$

If $\left(\tau_{1} f_{1}, \ldots, \tau_{p} f_{p}\right)$ is $V$-pseudo-invex and $\left(\lambda_{1} g_{1}, \ldots, \lambda_{m} g_{m}\right)$ is $V$-quasi-invex in nonsmooth sense, and $u$ is feasible in $(P)$, then $u$ is a global weak minimum of $(P)$. 
PROOF. Since $0 \in \sum_{i=1}^{p} \tau_{i} \partial f_{i}(u)+\sum_{j=1}^{m} \lambda_{j} \partial g_{j}(u)$, there exist $\xi_{i} \in \partial f_{i}(u)$ and $\zeta_{j} \in$ $\partial g_{j}(u)$ such that

$$
\sum_{i=1}^{p} \tau_{i} \xi_{i}+\sum_{j=1}^{m} \lambda_{j} \zeta_{j}=0 .
$$

Suppose that $u$ is not a global weak minimum point. Then, following the lines of proof of Theorem 3.1 of Jeyakumar and Mond [3], the $V$-pseudo-invexity conditions yield $\sum_{i=1}^{p} \tau_{i} \xi_{i} \eta\left(x_{0}, u\right)<0$. Thus, we have $\sum_{j=1}^{m} \lambda_{j} \zeta_{j} \eta\left(x_{0}, u\right)>0$. Then, $V$-quasiinvexity yields $\sum_{j=1}^{m} \beta_{j}\left(x_{0}, u\right) \lambda_{j} g_{j}\left(x_{0}\right)>\sum_{j=1}^{m} \beta_{j}\left(x_{0}, u\right) \lambda_{j} g_{j}(u)$. Since $x_{0}$ is feasible for $(\mathrm{P})$, that is, $\lambda_{j} g_{j}\left(x_{0}\right) \leq 0$, and $\lambda_{j} g_{j}(u)=0, j=1,2, \ldots, \lambda_{j}>0, \beta_{j}>0$. This contradicts the previous inequality.

For the problem (P), consider a corresponding Mond-Weir dual problem.

Maximise $\left(f_{i}(u): i=1,2, \ldots, p\right)$

subject to $\quad 0 \in \sum_{i=1}^{p} \tau_{i} \partial f_{i}(u)+\sum_{j=1}^{m} \lambda_{j} \partial g_{j}(u), \quad \lambda_{j} g_{j}(u) \geq 0, \quad j=1, \ldots, m$.

$$
\tau_{i} \geq 0, \quad \sum_{i=1}^{p} \tau_{i}=1, \quad \lambda_{j} \geq 0 .
$$

THEOREM 4.2 (Weak Duality). Let $X$ be feasible in $(\mathrm{P})$ and let $(u, \tau, \lambda)$ be feasible in (D). If $\left(\tau_{1} f_{1}, \ldots, \tau_{p} f_{p}\right)$ is $V$-pseudo-invex and $\left(\lambda_{1} g_{1}, \ldots, \lambda_{m} g_{m}\right)$ is $V$-quasi-invex as in Theorem 4.1, then $\left(f_{1}(x), \ldots, f_{p}(x)\right)^{\mathrm{T}}-\left(f_{1}(u), \ldots, f_{p}(u)\right)^{\top} \notin-$ int $R_{+}^{p}$.

PROOF. From the feasibility conditions, and $\beta_{j}(x, u)>0$, we have

$$
\sum_{j=1}^{m} \beta_{j}(x, u) \lambda_{j} g_{j}(x) \leq \sum_{j=1}^{m} \beta_{j}(x, u) \lambda_{j} g_{j}(u) .
$$

Then, by $V$-quasi-invexity, we have $\sum_{j=1}^{m} \zeta_{j} \eta(x, n) \leq 0, \forall \zeta_{j} \in \partial g_{j}(u)$. Since

$$
0 \in \sum_{i=1}^{p} \tau_{i} \partial f_{i}(u)+\sum_{j=1}^{m} \lambda_{j} \partial g_{j}(u),
$$

there exist $\xi_{i} \in \partial f_{i}(u)$ and $\zeta_{j} \in \partial g_{j}(u)$ such that $\sum_{i=1}^{p} \tau_{i} \xi_{i}+\sum_{j=1}^{m} \lambda_{j} \zeta_{j}(u)=0$. This implies that

$$
\sum_{i=1}^{p} \tau_{i} \xi_{i} \eta(x, n)+\sum_{j=1}^{m} \lambda_{j} \zeta_{j} \eta(x, u)=0 .
$$


Thus,

$$
\sum_{i=1}^{p} \tau_{i} \xi_{i} \eta(x, u) \geq 0, \quad \forall \xi_{i} \in \partial f_{i}(u) .
$$

The conclusion now follows from the $V$-pseudo-invexity condition since $\tau e=1$ and $\alpha(x, u)>0$.

THEOREM 4.3 (Strong Duality). Let $x^{0}$ be a weak minimum of $(\mathrm{P})$ at which a constraint qualification is satisfied. Then there exist $\tau^{0} \in R^{p}, \lambda^{0} \in R^{m}$ such that $\left(x^{0}, \tau^{0}, \lambda^{0}\right)$ is feasible in (D). If weak duality holds between (P) and (D), then $\left(x^{0}, \tau^{0}, \lambda^{0}\right)$ is a weak minimum of (D).

PROOF. From Kuhn-Tucker necessary conditions (see, for example, Theorem 6.1.3 of Clarke [1]), there exist $\tau \in R^{p}, \lambda \in R^{m}$ such that

$$
0 \in \sum_{i=1}^{p} \tau_{i} \partial f_{i}\left(x^{0}\right)+\sum_{j=1}^{m} \lambda_{j} \partial g_{j}\left(x^{0}\right),
$$

$\tau_{i} \geq 0, \tau \neq 0, \lambda_{j} \geq 0, \lambda_{j} g_{j}\left(x^{0}\right)=0, j=1,2, \ldots, m$. Now since $\tau_{i} \geq 0, \tau \neq 0$ we can scale the $\tau_{i}$ 's and $\lambda_{j}$ 's as

$$
\tau_{i}^{0}=\tau_{i} /\left(\sum_{i=1}^{p} \tau_{i}\right) \quad \text { and } \quad \lambda_{j}^{0}=\lambda_{j} /\left(\sum_{i=1}^{p} \tau_{i}\right) .
$$

Now we have $\left(x^{0}, \tau^{0}, \lambda^{0}\right)$ that is feasible in (D).

If $\left(x^{0}, \tau^{0}, \lambda^{0}\right)$ is not a weak maximum of (D), then there exists a feasible $(u, \tau, \lambda)$ for (D) such that

$$
\left(f_{1}(u), \ldots, f_{p}(u)\right)^{\top}-\left(f_{1}\left(x^{0}\right), \ldots, f_{p}\left(x^{0}\right)\right)^{\top} \in \text { int } R_{+}^{p} .
$$

Since $x^{0}$ is feasible in (P), this contradicts weak duality (Theorem 4.2).

\section{Nonsmooth multi-objective fractional programming}

In this section we apply the results of the previous section to study nonsmooth fractional multi-objective problems.

In the differentiable case, Jeyakumar and Mond [3] considered the fractional programming problem,

$$
V \text {-minimize }\left(\frac{p_{1}(x)}{q_{1}(x)}, \ldots, \frac{p_{r}(x)}{q_{r}(x)}\right)
$$


subject to $x \in X_{0}, g(x) \leq 0$, where $p_{i}: X_{0} \rightarrow R, q_{i}: X_{0} \rightarrow R$ and $g: X_{0} \rightarrow R^{m}$. It is assumed that $p_{i}(x) \geq 0$, for each $x$ on the feasible set $\Delta=\left\{x \in X_{0}: g(x) \leq 0\right\}$, $q_{i}(x)>0$, for each $x \in \Delta$. The problem (FI) is said to be a $V$-invex fractional problem if the functions $p, q$ and $g$ satisfy

$$
x, u \in \Delta \Rightarrow\left\{\begin{array}{l}
p_{i}(x)-p_{i}(u) \geq \gamma_{i}(x, u) p_{i}^{\prime}(u) \eta(x, u) \\
q_{i}(x)-q_{i}(u) \geq \gamma_{i}(x, u) q_{j}^{\prime}(u) \eta(x, u) \\
g_{j}(x)-g_{j}(u) \geq \beta_{j}(x, u) g_{j}^{\prime}(u) \eta(x, u)
\end{array}\right.
$$

with $\eta: X_{0} \times X_{0} \rightarrow R^{n}, \gamma_{i}, \beta_{j}: X_{0} \times X_{0} \rightarrow R_{+} \backslash\{0\}$.

Following Egudo and Hanson [2] we can generalise (FI) to the nonsmooth case by replacing $p_{i}^{\prime}, q_{i}^{\prime}$ and $g_{j}^{\prime}$ with the generalised gradients of Clarke. Hence (FI) is said to be $V$-invex nonsmooth fractional if there exists $\eta: X_{0} \times X_{0} \rightarrow R^{n}$ and $\gamma_{i}, \beta_{j}: X_{0} \times X_{0} \rightarrow R_{+} \backslash\{0\}$ such that for all $x, u \in \Delta$

$$
\begin{array}{rrr}
p_{i}(x)-p_{i}(u) \geq \gamma_{i}(x, u) \xi_{i} \eta(x, u), & \forall \xi_{i} \in \partial p_{i}(u), \\
q_{i}(x)-q_{i}(u) \leq \gamma_{i}(x, u) \zeta_{i} \eta(x, u), & \forall \zeta_{i} \in \partial q_{i}(u), \\
g_{j}(x)-g_{j}(u) \geq \beta_{j}(x, u) \mu_{j} \eta(x, u), & \forall \mu_{j} \in \partial g_{j}(u) .
\end{array}
$$

We need the following proposition from Clarke [1] in order to prove the main Theorem of this section.

PROPOSITION 5.1. (Clarke [1]). Let $f_{1}, f_{2}$ be Lipschitz near $x$, and suppose $f_{2}(x) \neq 0$. Then $f_{1} / f_{2}$ is Lipschitz near $x$, and

$$
\partial\left(\frac{f_{1}}{f_{2}}\right)(x) \subset \frac{f_{2}(x) \partial f_{1}(x)-f_{1}(x) \partial f_{2}(x)}{\left(f_{2}(x)\right)^{2}} .
$$

If in addition $f_{1}(x) \geq 0, f_{2}(x)>0$ and if $f_{1}$ and $-f_{2}$ are regular at $x$, then equality holds and $f_{1} / f_{2}$ is regular at $x$.

In the next theorem, we assume that $p_{1}$ and $p_{2}$ are regular.

THEOREM 5.1. Consider the problem (FI). Let $u \in \Delta$. Assume that there exist $(\tau, \lambda)$ such that $\tau \geq 0, \tau \neq 0, \lambda \geq 0$,

$$
0 \in \sum_{i=1}^{r} \tau_{i} \partial\left(\frac{p_{i}}{q_{i}}\right)(u)+\sum_{j=1}^{m} \lambda_{i} \partial g_{j}(u)
$$

and $\lambda_{j} g_{j}(u)=0, j=1,2, \ldots, m$. Then $u$ is a global weak minimum for $(\mathrm{FI})^{\prime}$. 
PROOF. The proof follows the lines of the proof of Theorem 4.1 of Jeyakumar and Mond [3] with appropriate changes in $\left(p_{i} / q_{i}\right)^{\prime}$. Proposition 5.1 plays a crucial role in this proof.

For a $V$-invex nonsmooth multi-objective fractional programming problem (FI)', the weak and strong duality properties hold with the following dual problem:

$$
\begin{array}{ll}
V \text {-maximise } & \left(\frac{p_{1}(u)}{q_{1}(u)}, \ldots, \frac{p_{r}(u)}{q_{r}(u)}\right) \\
\text { subject to } & 0 \in \sum_{i=1}^{r} \tau_{i} \partial\left(\frac{p_{i}}{q_{i}}\right)(u)+\sum_{j=1}^{m} \lambda_{j} \partial g_{j}(u) \\
& \lambda_{j} g_{j} \geq 0, \quad 1,2, \ldots, m \\
& \lambda_{j} \geq 0, \quad \tau \geq 0, \quad \tau e=1 .
\end{array}
$$

\section{Conclusion}

The Kuhn-Tucker subgradient conditions are shown to be sufficient for a weak minimum of a multi-objective programming problem when generalised invexity ( $V$ pseudo-invexity $/ V$-quasi-invexity) is present. Weak and strong duality theorems have been established. We use the results of Section 4 to extend Egudo and Hanson [2] to the fractional case in Section 5. If $p=1$, then our result extends the results on invexity used in Zhao [4] for the case of nonsmooth programming to pseudo-invexity and quasi-invexity.

\section{Acknowledgement}

The authors are indebted to Bruce Craven for his useful comments which led to the present improved version of the paper. The authors also wish to thank V. Jeyakumar and M. A. Hanson for sending their recent reprints and some of their unpublished works.

\section{References}

[1] F. H. Clarke, Optimization and nonsmooth analysis (Wiley-Interscience, New York, 1983).

[2] R. R. Egudo and M. A. Hanson, "On sufficiency of Kuhn-Tucker conditions in nonsmooth multiobjective programming", FSU Technical Report M-888, (1993). 
[3] V. Jeyakumar and B. Mond, "On generalised convex mathematical programming", J. Austral. Math. Soc. Ser. B 34 (1992) 43-53.

[4] F. Zhao, "On sufficiency of the Kuhn-Tucker conditions in non-differentiable programming", Bull. Austral. Math. Soc. 46 (1992) 385-389. 